\title{
ESTUDO DA RECRISTALIZAÇÃO SECUNDÁRIA DO AÇO SILÍCIO DE GRÃO ORIENTADO
}

\section{Resumo}

A forte orientação de Goss do aço silício de grão orientado é desenvolvida por recristalização secundária, cujos mecanismos são ainda objeto de estudo. Trabalhos recentes reforçam a teoria de que os contornos tipo CSL são responsáveis pelo crescimento anormal dos grãos com orientação de Goss. As estruturas de amostras de aço silício de grão orientado regular foram analisadas antes (estrutura primária) e após recristalização secundária (estrutura secundária). As características dos contornos formados pela orientação de Goss com as orientações da matriz primária foram determinadas. Os resultados mostraram que os contornos $\operatorname{CSL} \Sigma 5$ têm um papel importante na seleção dos grãos de Goss e que existe boa concordância entre as fraçôes de potenciais núcleos de Goss estimadas na matriz primária e as distribuições de orientação na estrutura secundária.

Palavras-chave: Aço silício de grão orientado; Recristalização secundária; Textura.

\section{GRAIN ORIENTED SILICON STEEL SECONDARY RECRYSTALLIZATION STUDY}

\section{Abstract}

The strong Goss orientation of grain oriented silicon steel is developed by secondary recrystallization. The mechanisms of abnormal grain growth in silicon steel are not completely understood. Recent publications suggest that CSL boundaries are responsible for the selectivity of Goss grains. In this paper the primary and secondary structures of regular grain oriented silicon steel samples were investigated. Boundary character of Goss grains with primary matrix orientations were determined. The results show that CSL $\Sigma 5$ boundaries play an important role in the selectivity of Goss grains and that there is good agreement between the fractions of potential Goss nuclei in the primary matrix and the orientation distribution in the secondary structure.

Key words: Grain oriented silicon steel; Secondary recrystallization; Texture.

\section{INTRODUÇÃo}

O aço silício de grão orientado é utilizado essencialmente na construção de núcleos de transformadores. $O$ produto comercial apresenta boas propriedades magnéticas na direção de laminação, devido a uma forte orientação preferencial (I I0)[00I], denominada orientação de Goss. A forte textura de Goss é desenvolvida por crescimento anormal dos grãos, ou recristalização secundária, a partir de uma matriz recristalizada, denominada matriz primária, onde o crescimento de grão normal é inibido por uma dispersão de partículas de precipitado.
Os mecanismos da recristalização secundária do aço silício são ainda objeto de estudo, não havendo um claro entendimento dos fatores que levam ao crescimento somente dos grãos com orientação de Goss. Os modelos propostos para explicar a recristalização secundária do aço silício de grão orientado são de dois tipos: nucleação seletiva e crescimento seletivo. Segundo o modelo da nucleação seletiva os núcleos seriam grãos com orientação de Goss, existentes na matriz primária, com vantagem de tamanho em relação à matriz( $(1)$. Faltam, entretanto, evidências experimentais que comprovem esta teoria. $O$ modelo do crescimento seletivo tem sido estudado recentemente e se baseia no fato de que os grãos com orientação de Goss têm maior probabilidade de formarem contornos especiais com as orientaçôes da matriz. Dois tipos de contornos têm sido estudados: contornos

\footnotetext{
I Pesquisador - Acesita S.A.

${ }^{2}$ Analista Consultor - Acesita S.A.
} 
tipo rede de pontos de coincidência( 2,3$)$ (coincidence site lattice CSL) e contornos de alta energia, com desorientação entre $20^{\circ}$ e $45^{\circ}$ (4). O crescimento seletivo dos grãos de Goss na recristalização secundária tem sido explicado com base na vantagem de mobilidade dos contornos especiais, $\operatorname{CSL}^{(2,3)}$ ou de alta energia, (4) ou com base na menor energia dos contornos tipo CSL.(5)

Alguns trabalhos recentes reforçam a teoria de que os contornos tipo CSL são responsáveis pelo crescimento anormal dos grãos com orientação de Goss.(6) Nos aços de grão orientado de alta permeabilidade, produzidos com apenas um estágio de laminação a frio e utiliza AIN e MgS como inibidor de crescimento de grão, o crescimento dos grãos de Goss tem sido atribuído aos contornos CSL $29 .(3,5)$ A maioria das investigaçôes descritas na literatura são realizadas com este tipo de aço. Nos aços de grão orientado regulares, produzidos com dois estágios de laminação a frio e utiliza apenas $\mathrm{MgS}$ como inibidor de crescimento de grâo, o crescimento dos grãos de Goss tem sido atribuído aos contornos CSL $\Sigma 5$.(7) Entretanto existem poucos estudos da recristalização secundária nos aços regulares. No presente trabalho são analisadas as estruturas primárias e secundárias de amostras de aço silício de grão orientado regular. Os resultados são analisados com base na teoria do crescimento seletivo em função dos contornos tipo CSL. Busca-se correlacionar parâmetros das estruturas primária e secundária.

\section{PROCEDIMENTO EXPERIMENTAL}

O material utilizado neste trabalho foi o aço silício de grão orientado regular, onde a inibição de crescimento normal de grão é realizada por uma dispersão de partículas de MnS. Amostras industriais laminadas a quente, na espessura de $2,45 \mathrm{~mm}$, foram processadas em laboratório com diferentes reduçốes nas duas etapas de laminação a frio. O processamento envolveu um recozimento inicial a $900^{\circ} \mathrm{C}$, laminação a frio, recozimento intermediário a $1030^{\circ} \mathrm{C}$, segunda etapa de laminaçâo a frio para espessura de $0,27 \mathrm{~mm}$, recozimento e descarbonetação a $900^{\circ} \mathrm{C}$ e recozimento final a $1200^{\circ} \mathrm{C}$, com aquecimento a $50^{\circ} \mathrm{C} / \mathrm{h}$. Foram laminadas três amostras, conforme reduçốes percentuais mostradas na tabela:

Tabela I. Reduçöes percentuais nas duas etapas de laminação a frio.

\begin{tabular}{ccc}
\hline Amostra & d Taminação & 2 Taminaçáo \\
\hline A & $75 \%$ & $56 \%$ \\
B & $70 \%$ & $63 \%$ \\
C & $63 \%$ & $70 \%$ \\
\hline
\end{tabular}

As estruturas obtidas após recozimento e descarbonetação a $900^{\circ} \mathrm{C}$, estrutura primária, e após o recozimento final a $1200^{\circ} \mathrm{C}$, estrutura secundária, foram analisadas por microscopia ótica e por EBSD. Nas análises de EBSD das estruturas primárias foram obtidos dados de pelo menos 6000 grãos por amostra. Nas estruturas secundárias, com textura forte e grãos muito grandes foram coletados em média 150 grãos por amostra. As propriedades magnéticas foram determinadas pelo teste Epstein ao longo da direção de laminação. Foram medidas a perda magnética a I,7T e $60 \mathrm{~Hz}\left(\mathrm{P}_{1,7}\right)$ e a polarização a $800 \mathrm{~A} / \mathrm{m}\left(\mathrm{B}_{8}\right)$.

\section{RESULTADOS E DISCUSSÃO}

A Figura I mostra as texturas após recozimento e descarbonetaçâo, para as três amostras, em seçôes no espaço de Euler para $\varphi_{2}=0^{\circ}$ e $\varphi_{2}=45^{\circ}$, notação de Bunge. Com a redução crescente na laminação a frio há um fortalecimento das fibras $\gamma$ e $\eta$, particularmente próximo a (III)[II2], (3I0)[00I] e (I00)[00I].

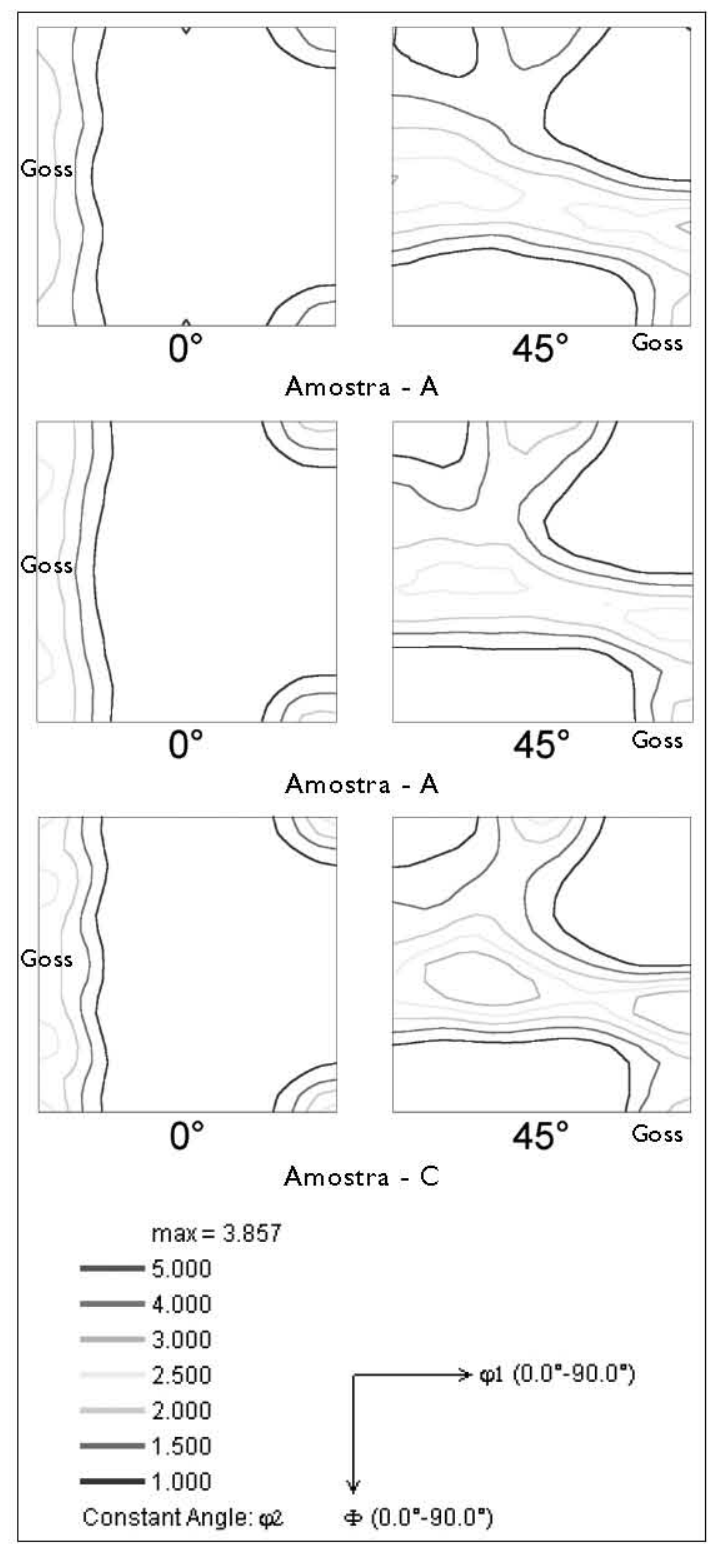

Figura I. Seçöes no espaço de Euler para $\varphi_{2}=0^{\circ}$ e $\varphi_{2}=45^{\circ}$ após recozimento e descarbonetação, estrutura primária, para as amostras A, B e C, respectivamente. 
As texturas após recristalização secundária são mostradas pelas figuras de polo $(00 \mathrm{I})$ da Figura 2. Na amostra A é observado o menor desvio médio da orientação ideal de Goss e na amostra $\mathrm{C}$ é observado o maior desvio. $\mathrm{Na}$ mesma figura são mostradas as macrografias correspondentes e os valores medidos de perda magnética $\left(\mathrm{P}_{1,7}\right)$ e polarização $\left(\mathrm{B}_{8}\right)$. As amostras $B$ e $C$ apresentarn grãos pequenos na estrutura, com elevado desvio da orientação de Goss, o que leva a menor permeabilidade e maior perda magnética final.

Para analisar as características dos contornos de grão formados pela orientação de Goss com as orientações da matriz primária foram calculadas as fraçôes de contornos CSL, de CSL $\Sigma 3$ a CSL $\Sigma 19 \beta$, entre a orientação de Goss e as orientações presentes na matriz primária, para as três amostras. Foi utilizado o critério de tolerância de Brandon. (II) Os resultados estão na Figura 3 e mostram que a maior fração é de contornos CSL $\Sigma 5$ e que esta fração é maior na amostra $A$ e menor na amostra $C$.

A orientação de Goss é a que tem maior fração de contornos $\Sigma 5$ com uma matriz primária túpica, embora não tenha a maior fração de contornos especiais, quando se considera o somatório das fraçôes de contornos CSL $\Sigma 3$ a $\Sigma 19 \beta$. Por matriz primária típica entende-se aquela que resulta, após recristalização secundária, em produto com pequeno desvio médio da orientação ideal de Goss, com polarização $\mathrm{B}_{8}$ da ordem de 1,87T.

Na Figura 4 são mostrados mapas das fraçōes de contornos CSL $\Sigma 5$ (figura 4a) e do somatório das fraçốes de contornos CSL $\Sigma 3$ a $\Sigma 19 \beta$ (figura 4b) formados numa matriz primária típica por orientaçôes presentes nas seçôes do espaço de Euler para $\varphi_{2}=0^{\circ}$ e $\varphi_{2}=45^{\circ}$. Para construção dos mapas na Figura 4 foram calculadas as fraçôes de contornos especiais para orientaçôes em intervalos de $\varphi_{\mathrm{I}}$ e $\phi$ de 5 em 5 graus. Os resultados da Figura 4a mostram que a maior parte dos contornos CSL $\Sigma 5$ são formados em torno dos grãos Goss da matriz primária e, por outro lado, a Figura $4 \mathrm{~b}$ indica que é mínima a incidência de contornos CSL com outros valores de sigma, em torno dos grãos Goss. Esses resultados indicam que os contornos CSL $\Sigma 5$ devam ser os responsáveis pela seleção dos grãos de Goss na recristalização secundária. Estes grãos têm a maior probabilidade de formar contornos CSL $\Sigma 5$ com a matriz primária.
Para que um grão com orientação de Goss seja um núcleo para recristalização secundária ele deve fazer contornos especiais CSL $\Sigma 5$ com grãos vizinhos. Devido à menor energia dos contornos CSL $\Sigma 5$ a força de arraste exercida pelas partículas sobre os mesmos será menor que sobre os demais contornos. Durante o recozimento final as partículas de precipitado tenderão a coalescer, reduzindo a força de arraste. Os contornos de menor energia migrarão antes dos demais, permitindo o crescimento dos núcleos de Goss.

A fração de potenciais núcleos de Goss depende de dois fatores: da fração de contornos CSL $\Sigma 5$ formados pela orientação de Goss, ou orientaçôes próximas, com a matriz primária $\left[\Sigma_{5}(G)\right]$; e da fração de grãos com orientação de Goss, ou próxima da

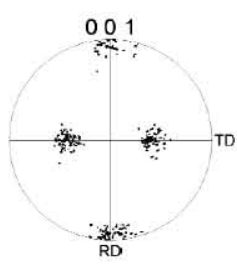

(a)

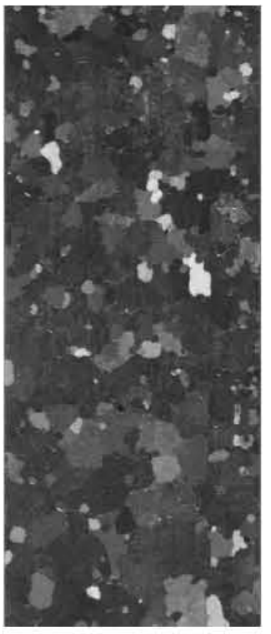

(d)

$$
\begin{aligned}
\mathrm{P}_{1,7} & =1,528 \mathrm{~W} / \mathrm{kg} \\
\mathrm{J}_{8} & =1,836 \mathrm{~T}
\end{aligned}
$$

$\mathrm{J}_{8}=1,805 \mathrm{~T}$ após recristalizaçäo secundária: $d, e, f$ macrografias das estruturas finais, para as amostras $\mathrm{A}, \mathrm{B}$ e $\mathrm{C}$, respectivamente.

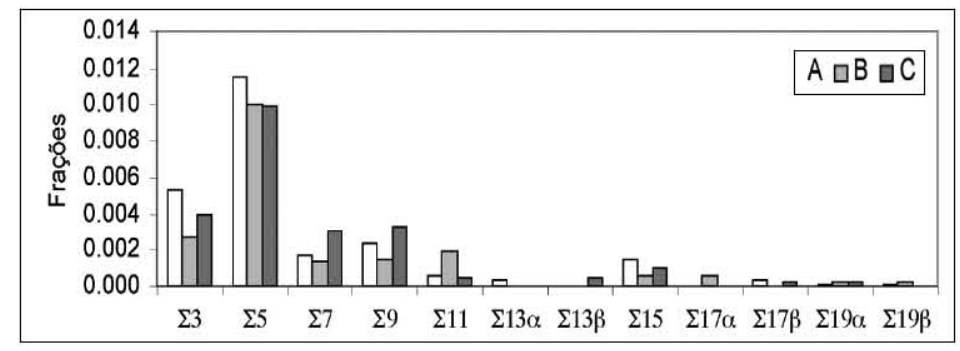

Figura 3. Fraçōes de contornos CSL até $\Sigma \mid 9 b$ nas amostras $A, B$ e $C$. 


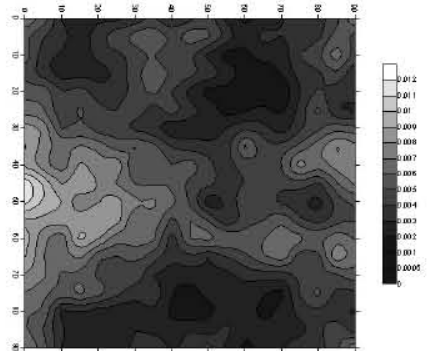

CSL $\Sigma 5 \quad \varphi_{2}=0^{\circ}$

(a)

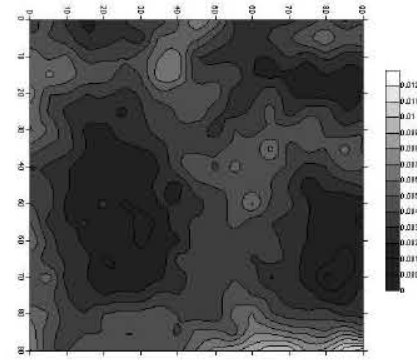

$\operatorname{CSL} \Sigma 5 \varphi_{2}=45^{\circ}$

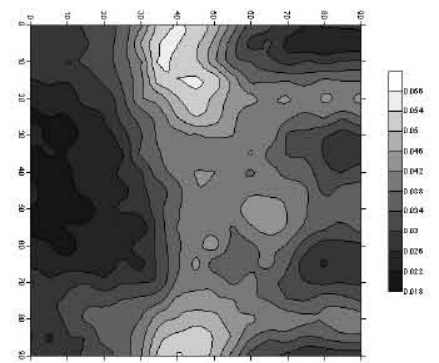

$\operatorname{CSL} \Sigma 3$ a $\Sigma 19 \beta \quad \varphi_{2}=0^{\circ}$

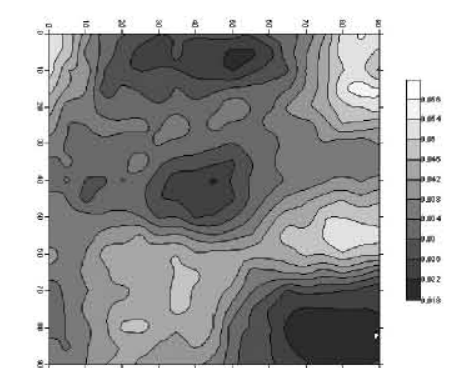

(b)
$\operatorname{CSL} \Sigma 3$ a $\Sigma 19 \beta \quad \varphi_{2}=45^{\circ}$

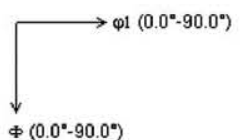

Figura 4. Mapas das fraçöes de contornos (a ) CSL $\Sigma 5$ e (b ) do somatório das fraçöes de contornos CSL $\Sigma 3$ a $\Sigma 19 \beta$ formados numa matriz primária típica por orientaçöes presentes nas seçöes do espaço de Euler para $\varphi_{2}=0^{\circ}$ e $\varphi_{2}=45^{\circ}$.

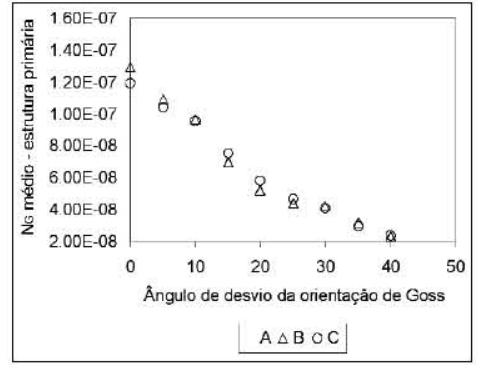

(a)

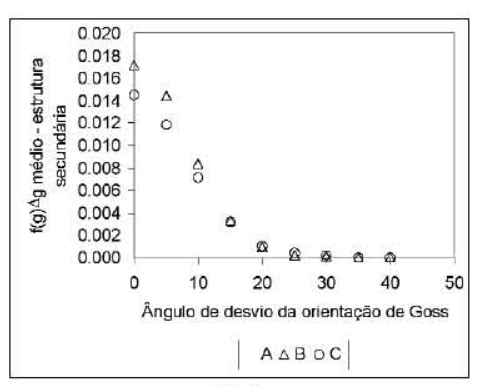

(b)

Figura 5. a) Fraçōes médias de potenciais núcleos, na estrutura primária, para desvios da orientação ideal de Goss em torno das direçöes DL, DT, DN; b) valores médios de $f(g) \Delta g$ da estrutura secundária, para desvios da orientação ideal de Goss em torno de DL, DT, DN.

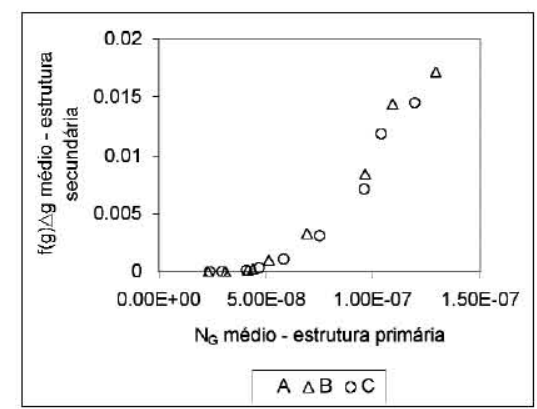

Figura 6. Valores médios de $f(g) \Delta g$ da estrutura secundária em função das fraçōes médias de potencias núdeos na estrutura primária, medidos para desvios da orientaçäo ideal de Goss até $40^{\circ}$. Os valores säo médias obtidos para desvios em torno das direçöes DL, DT, DN.

orientação ideal, na matriz primária. O segundo fator pode serestimado a partir da função distribuição de orientação cristalográfica (FDOC) pelo produto $\mathrm{f}(\mathrm{g}) \Delta \mathrm{g}$. Assim, a fração de potenciais núcleos pode ser estimada pelo produto:

$N_{G}=\Sigma_{5}(G) f(g) \Delta g \quad$ Onde $\Delta g=\frac{1}{8 \pi^{2}} \cos \phi \Delta \varphi_{1} \Delta \phi \Delta \varphi_{2}$

Para estimar as frações de potenciais núcleos foram calculados valores de $\mathrm{N}_{G}$ para orientações até $40^{\circ}$ da orientação ideal de Goss, em torno dos eixos paralelos às direçốes de laminaçâo (DL), transversal (DT) e normal (DN). Os valores médios das três direçôes $\left(\overline{N_{G}}\right)$ estão mostrados na Figura 5 a para as amostras A, $\mathrm{B}$ e $\mathrm{C}$.

Observa-se que a fração de potenciais núcleos de Goss $\left(\overline{N_{G}}\right)$ decresce com o aumento do desvio da orientação ideal e que para pequenos desvios os valores da amostra $\mathrm{A}$ são maiores, seguidos das amostras B e C, respectivamente. A amostra $\mathrm{C}$ tem maiores valores de $\overline{N_{G}}$ na faixa de desvios de $15^{\circ}$ a $25^{\circ}$. Para comparar estes resultados com as distribuiçôes de orientação nas estruturas secundárias foram calculados os valores de $f(g) \Delta g$, para os mesmos desvios da orientação de Goss, em torno das direçôes DL, DT e DN. Os valores médios obtidos para as estruturas secundárias estão mostrados na Figura 5b. Existe uma concor dância razoável entre as distribuições das fraçôes de potenciais núcleos nas estruturas primárias e as distribuiçôes de orientação nas estruturas secundárias. Estes resultados reforçam a teoria do crescimento seletivo baseado em contornos CSL e confirmam que os contornos CSL $\Sigma 5$ têm um papel importante no processo de seleção dos grãos de Goss na recristalização secundária do aço silício de grão orientado regular.

Na Figura 6 são mostrados valores médio de $f(g) \Delta g$ das estruturas secundárias em função das fraçôes médias de núcleos potenciais $\left(\overline{N_{G}}\right)$ das estruturas primárias. Os dados sugerem que existe uma fração média mínima para que ocorra o crescimento dos grãos na recristalização secundária, da ordem de $10^{-7}$, este valor esta próximo do número de grãos da matriz engolidos pelo grão Goss no crescimento anormal, da ordem de $10^{6}$. 


\section{CONCLUSÓES}

Contornos CSL $\Sigma 5$ têm um papel importante na seleção dos grãos de Goss na recristalização secundária do aço silício de grão orientado regular. A fração de potenciais núcleos de Goss na estrutura primária pode ser estimada em função da fração de contorno CSL $\Sigma 5$ e da distribuição de orientaçẫo na estrutura primária. Existe boa concordância entre as fraçōes de potenciais núcleos de Goss e a distribuição de orientaçōes na estrutura secundária.

\section{REFERÊNCIAS}

I BRANDON, D. G. The structure of high angle grain boundaries. Acta Metallurgica, v. 14, p. 1479- 1483, 1966.

2 HARASE, J.; SHIMIZU, R.; WATANABE, T. The effects of the grain boundary character distribution on grain growth ad texture formation in Fe-3\%Si. In: RISOE INTERNATIONAL SYMPOSIUM ON METERIALS MATERIALIA SCIENCE, 7., 1986, Alemanha. Proceedings... Llcotom 7 : H.J. Bunge, 1986. p. 343-348.

3 SHIMIZU, R.; HARASE, J.; DINGLEY, D.J. Prediction of secondary recrystallization texture in Fe-3\%Si by three dimensional texture analysis. Acta Metallurgica Materialia, v. 38, n.6, p. 973-978, Jun. 1990.

4 HAYAKAWA, Y.; SZPUNAR, J.A. The role of grain boundary character distribution in secondary recrystallization of electrical steels, Acta Materialia, v. 45, n.3, p. 1285-1295, Mar. 1997.

5 USHIGAMi, Y.; KAWASAKI, K.; NAKAYAMA, T.; SUGA, Y.; HARASE, J.; TAKAHASHI, N. Dynamic observation of the growth of secondary recrystallization grains of $\mathrm{Fe}-3 \% \mathrm{Si}$ alloy utilizing synchrotron $\mathrm{x}$-ray topography. Materialia Science Forum, v. 157-162, p. 108I-1085, Ago. 1994.

6 NAKAMURA, S.; USHIGAMI, Y.; TAKEBAYASHI, S.; SUZUKI, S. Coincidence grain boundary and selective growth of goss grain in Fe-3\%Si alloy. Materialia Science Forum, v. 408-4I2, p. 1293-1298, Sept. 2002.

7 LEE, K.T.; SZPUNAR, U.A. The role of special grain boundaries during the grain growth in Fe-3\%Si. Canadian Metallurgica Quarterly, v. 34, n.3, p.257-263, Oct. 1995.

Recebido em: 09/12/05

Aceito em: 15/03/06

Proveniente de: CONGRESSO ANUAL DA ABM, 60., 2005, Belo Horizonte. São Paulo : ABM, 2005. 